\title{
Multi-Tap Photonic Microwave Filter Based on Two-Pump Fiber Optical Parametric Amplifier
}

\author{
Jia Li, Kim K. Y. Cheung, Xing Xu, and Kenneth K. Y. Wong* \\ Photonic Systems Research Laboratory, Department of Electrical and Electronic Engineering, \\ The University of Hong Kong, Pokfulam Road, Hong Kong \\ *Email: kywong@eee.hku.hk
}

\begin{abstract}
A multi-tap photonic microwave filter based on two-pump fiber optical parametric amplifier (OPA) is proposed and an 8-tap filter is experimentally demonstrated. Tunability of the filter is also investigated in the paper, which shows consistency between experimental and theoretical results.

(C2009 Optical Society of America

OCIS codes: (190.4970) parametric oscillators and amplifiers; $(060.5625)$ radio frequency photonics
\end{abstract}

\section{Introduction}

Photonic microwave filter attracts more interests recently in the broadband wireless access networks as well as radar and photonic beamsteering of phased-arrayed antennas because of its inherent advantages such as low loss, high bandwidth, immunity to electromagnetic interference (EMI) compared to the ordinary microwave filters [1].

Basically, photonic microwave filters are implemented based on delay-line structure where multiple laser sources are usually used in order to achieve a linear relationship between the input and output radio-frequency (RF) signal and to enhance the Q-factor of the filter. Several approaches have been proposed [2-4]. However, in these approaches the number of laser sources required equals to the number of filter taps, which increases the cost of the system if multi-tap filter is implemented. Recently, a one-pump fiber OPA based photonic microwave filter is demonstrated which can reduce the number of laser sources, but requires a complex delay-line structure [5]. In this paper, we propose a novel method of using two-pump OPA to construct photonic microwave filter which has a much simpler delay-line structure providing flexible tuning ability, and to reduce the laser sources by almost half.

\section{Principle}

The principle of two-pump OPA based photonic microwave filter is shown in Fig. 1 (a). Two strong continuouswave (CW) pumps propagate along a spool of nonlinear fiber. The center wavelength of the two pumps is located around the zero-dispersion wavelength $v_{0}$ of the nonlinear fiber such that a wide range of signal wavelengths can meet the phase-matching conditions. The wavelengths and powers of the two pumps are carefully chosen to ensure the flatness of the gain spectrum [6]. Then if multiple signal wavelengths are launched into the nonlinear fiber with their wavelengths in the gain region, the pumps will transfer part of their energy to the signals and at the same time to generate another set of wavelengths which are called idlers as shown in Fig. 1 (b). The wavelengths of the signals and idlers are symmetric with respect to the center of the two pumps in terms of frequency. So it is possible to obtain the signals and idlers with equally-spaced wavelengths by careful selection of the signal wavelengths. Then all the signals and idlers are selected using a tunable bandpass filter (TBPF) and launched into a spool of dispersive fiber to introduce an equal amount of time delay between adjacent channels. Time-delayed optical signals are then detected and converted back into electrical signal in the photodetector (PD).
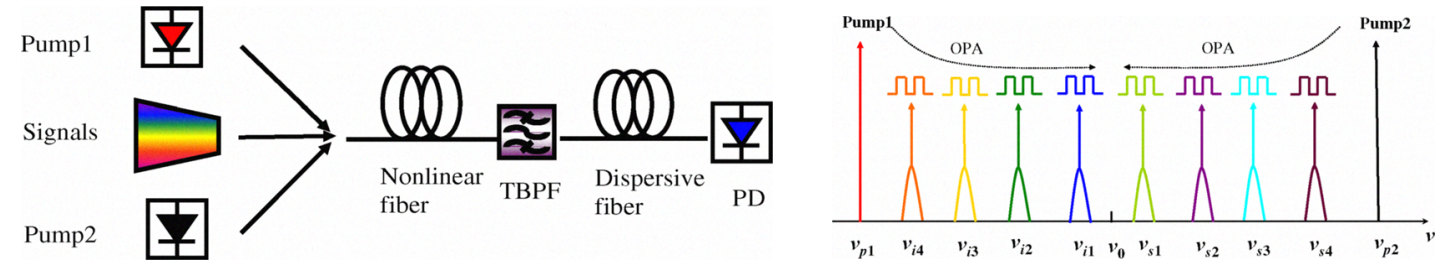

Fig. 1 (a) Two-pump OPA based photonic microwave filter. (b) Principle of two-pump OPA. $v_{p}$ : pump waves, $v_{i}$ : idler waves, $v_{s}$ : signal waves.

\section{Experiment}

The experimental setup is shown in Fig. 2. Two tunable laser sources (TLSs) TLS1 and TLS2 serve as two pump waves with wavelength of $1535 \mathrm{~nm}$ and $1547 \mathrm{~nm}$, respectively. They are then combined together using a 50/50 optical coupler (OC) and launched into a phase modulator (PM). The two pumps are phase modulated by $10-\mathrm{Gb} / \mathrm{s}$ $2^{7}-1$ pseudo-random binary sequence (PRBS) to suppress stimulated Brillouin scattering (SBS). After phase dithering the two pumps are splitted into two paths by a wavelength-division multiplexing coupler (WDMC1) and 


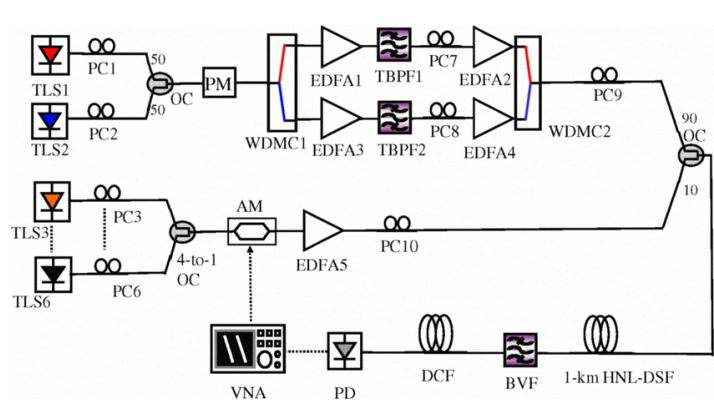

Fig. 2 Experimental setup for two-pump OPA-based photonic microwave filter. Refer to text for details.

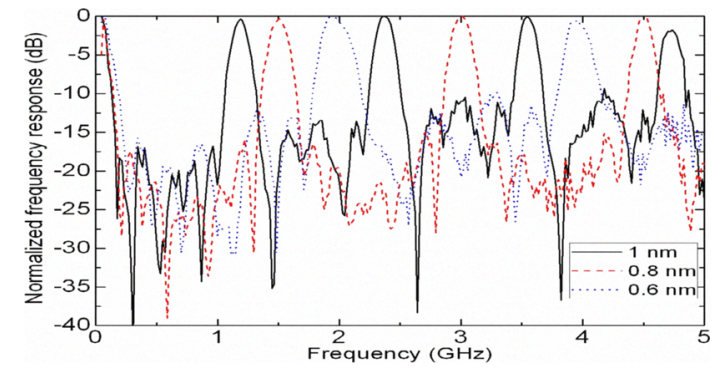

Fig. 3 Frequency response of 8-tap filter. Black solid, red dashed, and blue dotted lines corresponds to wavelength separation of $1 \mathrm{~nm}, 0.8 \mathrm{~nm}$, and $0.6 \mathrm{~nm}$, respectively.

amplified by two-stage erbium-doped fiber amplifiers (EDFAs) with TBPFs between them to suppress amplified spontaneous emission (ASE) noise. Each pump is amplified to a power of $22 \mathrm{dBm}$. Polarization controllers (PCs) PC7 and PC8 are used to adjust the state of polarization (SOP) of the two pumps to be orthogonal so that the crossgain modulation (XGM) and four-wave mixing (FWM) effects can be reduced [7]. They are then combined using another WDMC2. As for the signal branch, TLS1-4 corresponds to the four input signals. They are combined together using a 4-to-1 OC and launched into an amplitude modulator (AM). Optical power of each channel is -12 $\mathrm{dBm}$ before the nonlinear fiber. Then signals and pumps are combined together using a 10/90 OC. They are then launched into a spool of highly-nonlinear dispersion-shifted fiber (HNL-DSF) with the zero-dispersion wavelength of $1542 \mathrm{~nm}$ and the nonlinear coefficient of $10.4 \mathrm{~W}^{-1} \mathrm{~km}^{-1}$. After parametric amplification, four signals and four idlers are selected by a bandwidth-variable tunable filter (BVF). Then they propagate along a spool of dispersioncompensating fiber (DCF) with dispersion of $850 \mathrm{ps} / \mathrm{nm}$ to introduce a relative time delay between eight channels. The frequency response of the filter is monitored by the vector network analyzer (VNA) after photodetection.

Fig. 3 shows the experimental results. Firstly, the wavelengths of the four signals are set to be $1537.5 \mathrm{~nm}, 1538.5$ $\mathrm{nm}, 1539.5 \mathrm{~nm}$, and $1540.5 \mathrm{~nm}$, so the wavelength separation is $1 \mathrm{~nm}$. As a result of dispersion induced time delay, the temporal separation of adjacent channels is $850 \mathrm{ps}$, corresponding to a free spectral range (FSR) of $1.18 \mathrm{GHz}$, which matches with the experimental result shown in the black solid line of Fig. 3. Then we change the wavelength separation from $1 \mathrm{~nm}$ to $0.8 \mathrm{~nm}$ and $0.6 \mathrm{~nm}$, the frequency response curve is observed to shift to a higher frequency as shown in the red dashed line and blue dotted lines of Fig. 3. The FSR also changes from $1.18 \mathrm{GHz}$ to $1.50 \mathrm{GHz}$ and $2 \mathrm{GHz}$, respectively, which also agrees well with the theoretical value. A mainlobe-to-sidelobe ratio (MSR) of more than $10 \mathrm{~dB}$ is obtained in the experiment. The wavelength separation is changed by simply changing the four signals' wavelengths without changing the other parameters of the system. So the proposed photonic microwave filter is tunable and a multi-tap configuration can be implemented using less laser sources.

\section{Conclusions}

We proposed and demonstrated a novel photonic microwave filter based on two-pump fiber OPA. In this scheme, only half of the laser sources are required to achieve a multi-tap operation. The filter is also tunable by simply tuning the wavelengths of the signals, which shows consistency between experimental and theoretical results.

\section{Acknowledgment}

The work described in this paper was partially supported by a grant from the Research Grants Council of the Hong Kong Special Administrative Region, China (Project No. HKU 7172/07E and HKU 7179/08E). The authors would also like to thank Alnair Labs for providing the BVF.

\section{References}

[1] R. A. Minasian, "Photonic signal processing of microwave signals," IEEE Trans. Microw. Theory Tech. 54, 832-846 (2006).

[2] J. Capmany, D. Pastor, and B. Ortega, "New and flexible fiber-optic delay-line filters using chirped Bragg grating and laser arrays," IEEE Trans. Microwav. Theory Tech., 47, 1321-1326 (1999).

[3] V. Polo, B. Vidal, J. L. Corral, and J. Marti, "Novel tunable photonic microwave filter based on laser arrays and N $\times$ N AWG-based delay lines," IEEE Photon. Tech. Lett., 15, 584-586 (2003).

[4] Q. Wang, and J. P. Yao, "Multitap photonic microwave filters with arbitrary positive and negative coefficients using a polarization modulator and an optical polarizer," IEEE Photon. Technol. Lett. 20, 78-80 (2008)

[5] J. Li, K. K. Y. Cheung, and K. K. Y. Wong, "Photonic microwave filter with negative coefficients using fiber optical parametric amplifier," in Proc. OFC/NFOEC 2009, paper JWA53 (2009).

[6] M. E. Marhic, Y. Park, F. S. Yang and L. G. Kazovsky, "Broadband fiber-optical parametric amplifiers and wavelength converters with lowripple Chebyshev gain spectra," Opt. Lett., 21, 1354-1356 (1996).

[7] K. K. Y. Wong, G. W. Lu, and L. K. Chen, "Experimental studies of the WDM signal crosstalk in two-pump fiber optical parametric amplifiers,” Opt. Commun., 270, 429-432 (2007). 